\title{
Peregrine falcon wakes examined using Volumetric PIV
}

\section{Chetan Jagadeesh¹, Edward Talboys ${ }^{1}$, Daniel Troolin ${ }^{2}$, Martin Hyde ${ }^{2}$}

${ }^{1}$ City, University of London, Aeronautics and Aerospace Research Centre, London, United Kingdom

2TSI Incorporated, Fluid Mechanics Research Instruments, St. Paul, MN, USA

*dtroolin@tsi.com

\begin{abstract}
This study presents time-resolved volumetric measurements in the wake of a peregrine falcon model. The experiments were performed in a water flume with a freestream velocity of $10 \mathrm{~cm} / \mathrm{s}$ and at an angle of $3.25^{\circ}$. The TSI volumetric PIV system, using Insight V3V-4G software, was used to capture the time-resolved volumetric flow field. The results compare well with previous Stereo PIV measurements; however, the present results also provide true 3-dimensional flow field information which helps decode the reason for the superior maneuverability. This is attributable to the vortex dominated flow field promoted by its morphology.
\end{abstract}

\section{$1 \quad$ Introduction}

The peregrine falcon (Falco peregrinus) is well known to be the fastest avian species and aerodynamicists have been looking as to how these birds can achieve the speeds they have been observed to achieve in nature. When the bird is in its stoop, it increases speed by furling its wings up such that it is in its most aerodynamic form; named the Teardrop configuration. Once the falcon needs to control the stoop it opens its wings into the 'M-shape' where the bird has its most control. This is the configuration which is studied further herein. In a previous study (Gowree et al., 2018) it was observed that the aerodynamics of the falcon is dominated by complicated vortical structures over the bird. In the present work we will be examining in more detail the vortices that are induced by the tip of the wings, named the 'wing vortex' and 'primary feather vortex'. More recently Selim et al., 2021 has built upon the results of Gowree et al. by studying the stability of the falcon during the stoop phase. They report that when the falcon deploys the hand-wing, in the pull-out phase of the stoop, it creates extra vortex lift in a similar manner to combat aircraft. They also concluded that the falcon maximizes responsiveness by flying in a marginally longitudinally unstable mode where small changes in the hand-wings are used to help the bird stay in control giving them a competitive aerodynamic advantage over their prey.

\section{Experimental Setup}

A TSI volumetric particle image velocimetry (VPIV) system was used to acquire velocity fields downstream of a peregrine falcon model in a water flume. The falcon model was manufactured using a Polyjet 3D printer and possessed approximate dimensions of $0.3 \mathrm{~m}$ by $0.17 \mathrm{~m}$. The water flume test section was $1.4 \mathrm{~m} \times 0.4 \mathrm{~m} \times 0.45 \mathrm{~m}$, and the falcon model was placed $0.4 \mathrm{~m}$ from the inlet and along the centerline of the test section. 
The experimental setup can be seen in Fig. 1. Four Phantom M310 cameras were arranged on a rail in a line configuration at angles from the normal of the side-wall of the flume of approximately $-16^{\circ}$, $7^{\circ}, 3^{\circ}$, and $12^{\circ}$, and labeled cameras $1,2,3$, and 4 , respectively. Three of the cameras were fitted with $100 \mathrm{~mm}$ lenses and camera 3 (one of the inner cameras) was fitted with a $105 \mathrm{~mm}$ lens. The outer two cameras ( 1 and 4 ) were also fitted with Scheimpflug mounts in order to correct for distortion effects associated with viewing the measurement volume through a refractive index change at a larger angle.

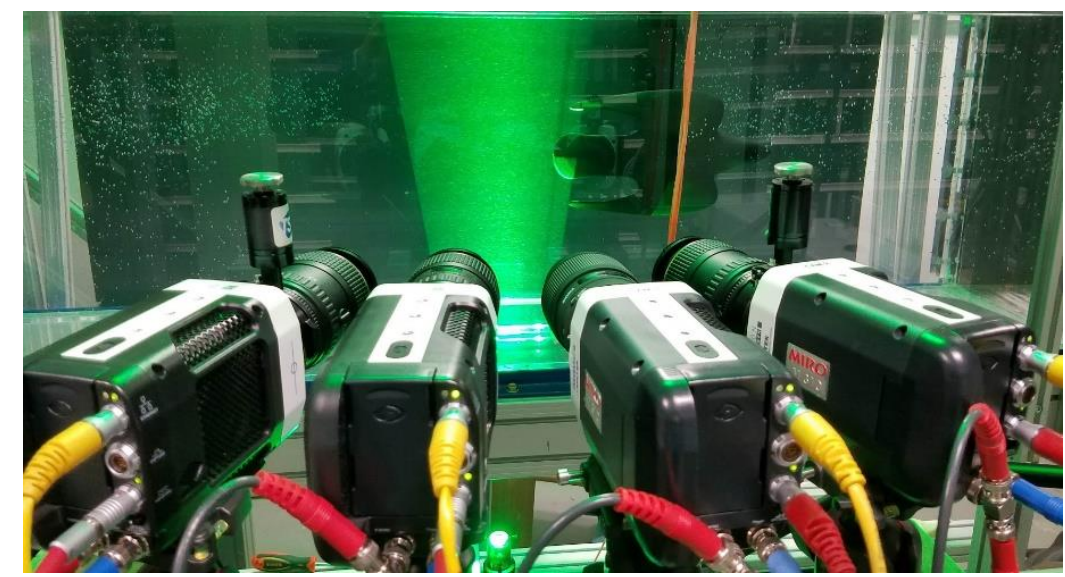

Fig. 1: Experimental setup showing the position of the four cameras and the laser measurement volume downstream of the falcon model (flow is from right to left in this image).

A Litron LDY300 dual-head laser which produced approximately $20 \mathrm{~mJ} /$ pulse was used to illuminate the measurement volume. A light arm was positioned at the exit of the laser in order to direct the laser light beneath the water channel. Light volume optics including a $-25 \mathrm{~mm}$ cylindrical lens and an adjustable focal length spherical lens were used to direct the illumination cone of laser light up through the transparent bottom window of the channel illuminating the measurement volume of size $115 \times 68 \times 17.5 \mathrm{~mm}$.

Data was acquired at a freestream flow velocity of nominally $10 \mathrm{~cm} / \mathrm{s}$ with the falcon model positioned at the centerline of the water channel at an angle of attack of $3.25^{\circ}$ from the streamwise direction. The center of the measurement volume was located $80 \mathrm{~mm}$ downstream of the wing-tip. Data was taken at three separate positions along the span of the channel, offset by $10 \mathrm{~mm}$ each. The result was three volumes that were overlapped with the neighboring volume by approximately $5 \mathrm{~mm}$. The position of the measurement volumes can be seen in Fig. 2. 


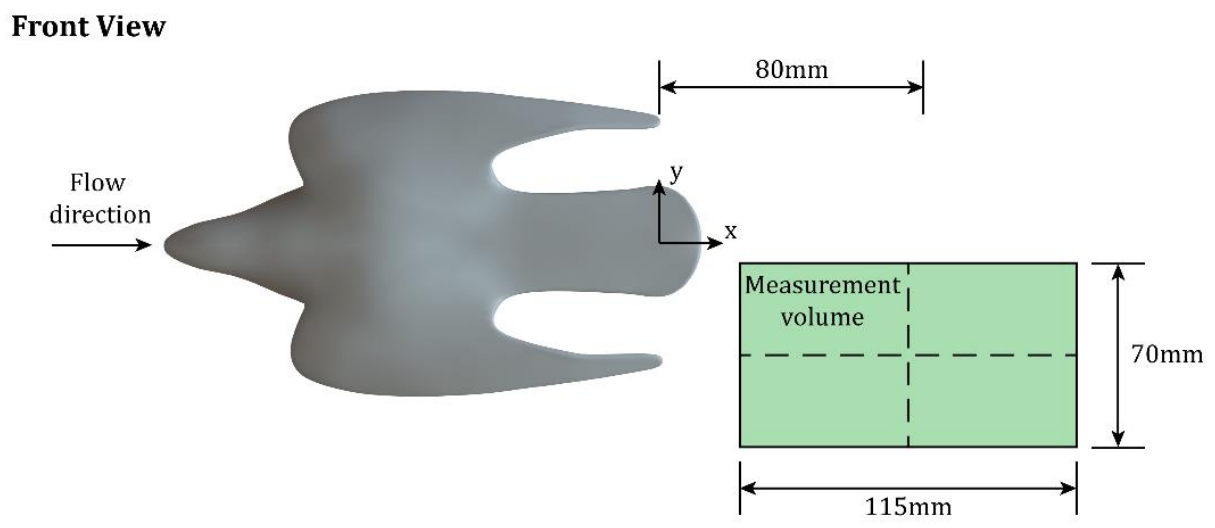

Top View

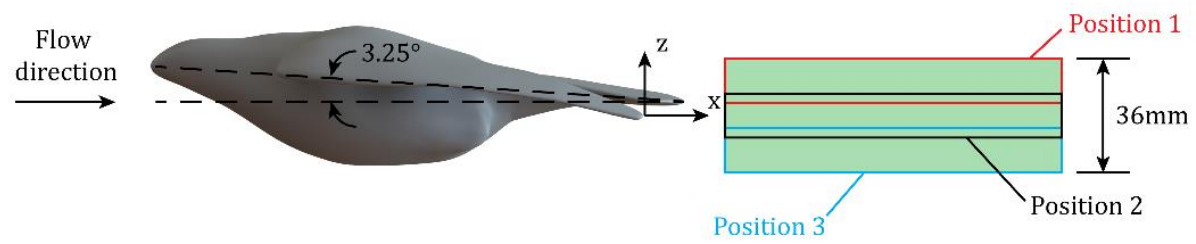

Fig. 2: Schematic representation of the measurement volumes with respect to the falcon model.

The images were captured at a frequency of $400 \mathrm{~Hz}$, or $2.5 \mathrm{~ms}$ between images. The high capture rate allowed for tracking between frames separated by up to $6^{*} \mathrm{dT}$, or $15 \mathrm{~ms}$. A benefit of this oversampling was that it allowed for a rolling ensemble of particle positions from 16 temporal realizations to be combined in order to achieve high spatial and temporal resolution measurements.

InsightV3V-4G software was used to coordinate the laser pulses and image capture timing as well as for performing the particle tracking and data analysis. For each experimental run, 2000 image pairs were acquired and processed. Data vector processing was performed using dense particle identification and reconstruction (DPIR), a technique detailed in Boomsma and Troolin, 2018. Both ensemble and instantaneous velocity fields were calculated and examined.

\section{$3 \quad$ Results and Discussion}

Particle track positions consisting of 20 time-steps and colored by the streamwise velocity can be seen in Fig. 3a. A region of blue stretching from the left side toward the right indicates the slower moving wake downstream of the wing-tip generating vorticity and vortex shedding that can be inferred from the alternating red/green coloring above the blue wake.

Figure $3 \mathrm{~b}$, shows the average streamwise velocity, with an isosurface of Q-criterion. This isosurface indicates the position of the core of the vortex from the wing tip vortex. From the end plane, the wake deficit can be clearly seen from the model of the bird. 

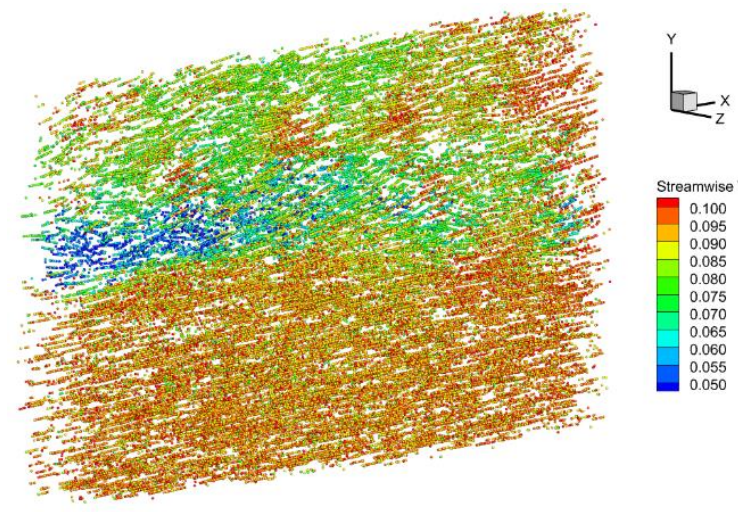

(a) Particle tracks for the wake downstream of the falcon model. Flow is from left to right and into the page ( $\mathrm{x}$ axis).

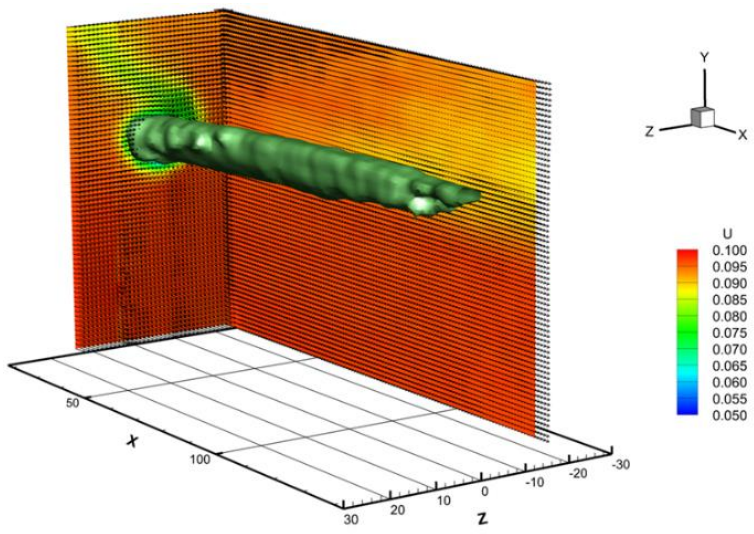

(b) Ensembled average of the three measurement zones. The contours show the streamwise velocity and the iso-surface shows the $\mathrm{Q}$-criterion at $\mathrm{Q}=3 \mathrm{sec}^{-1}$.

Fig. 3: Results of VPIV experiments

The plot in Fig. 4 is an instantaneous velocity field at position 2 . This section is one where the vortex core was present. Here, the slices represents streamwise velocity, and the red isosurfaces represent the $Q$ criterion a $Q=3 \mathrm{sec}^{-1}$. In this snapshot $Q$ shedding from the wing tip and propagating within the wake is clearly seen in the red isosurfaces that span from the left to the right as they convect downstream.

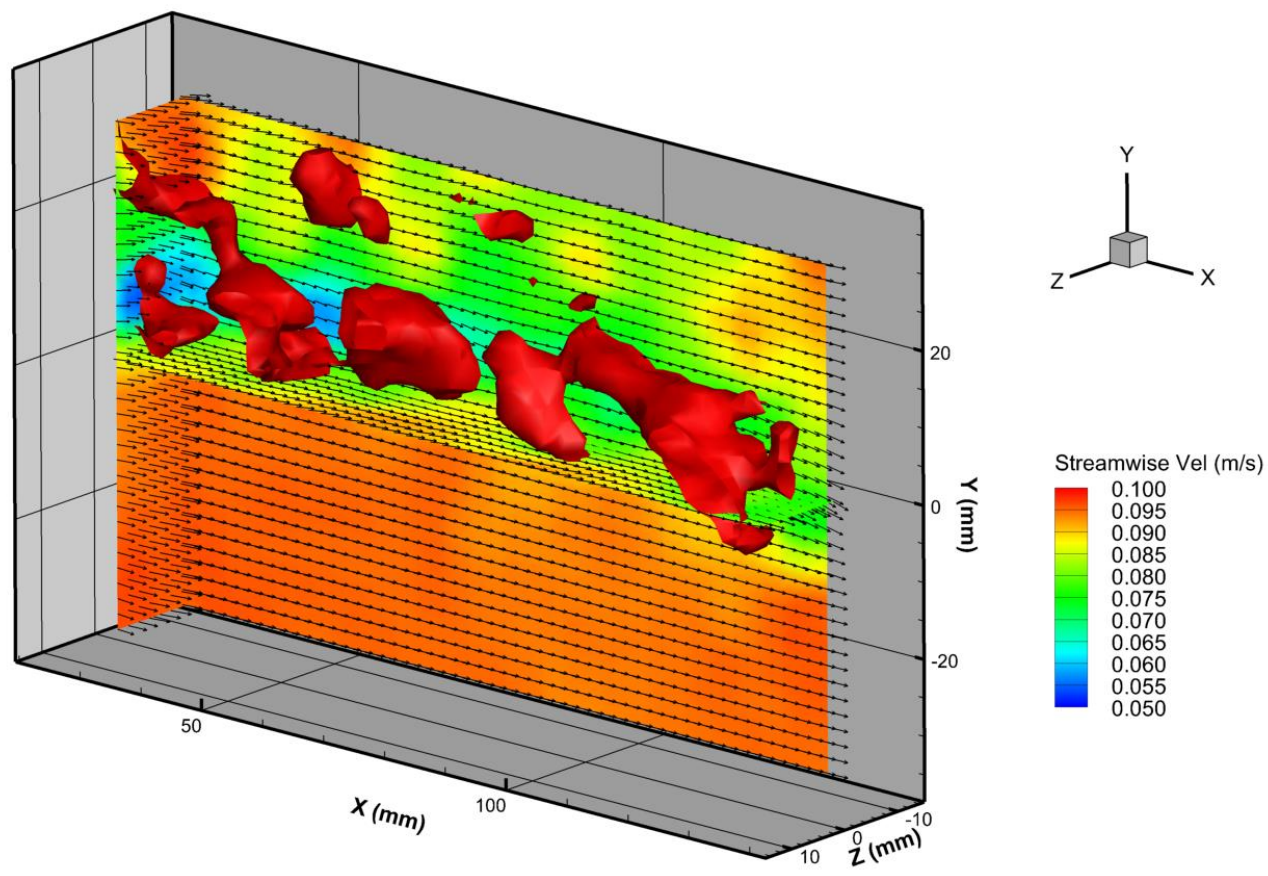

Fig. 4: Instantaneous velocity field showing the streamwise velocity as well as an iso-surface of the $Q$-criterion at a value of $Q=3 \sec ^{-1}$. 
Fig. 5 shows a comparison between the averaged non-dimensional x vorticity from this study and the study carried out on the same falcon model, where the authors only used SPIV. The differences in the two studies are that the flow medium was different, here was in a water tunnel and in Gowree et al. was in a wind tunnel. As such the x-vorticity was non-dimensionalised with the respective freestream velocity and length scale (the length of the bird model). It can be seen that the magnitudes of the two contour levels are not quite the same as each other and is thought to be due to a slightly increased angle of incidence in the Gowree study $\left(\alpha=5^{\circ}\right)$. However, the general positioning and structure of the two vortices are the same. This gives confidence in the current experimental set-up, such that it could be used for future research using the VPIV to allude more details into the complex vortex driven flow of the peregrine falcon. It also gives confidence in the processing algorithms to accurately calculate and track the particles in a complex vortex driven flow field.

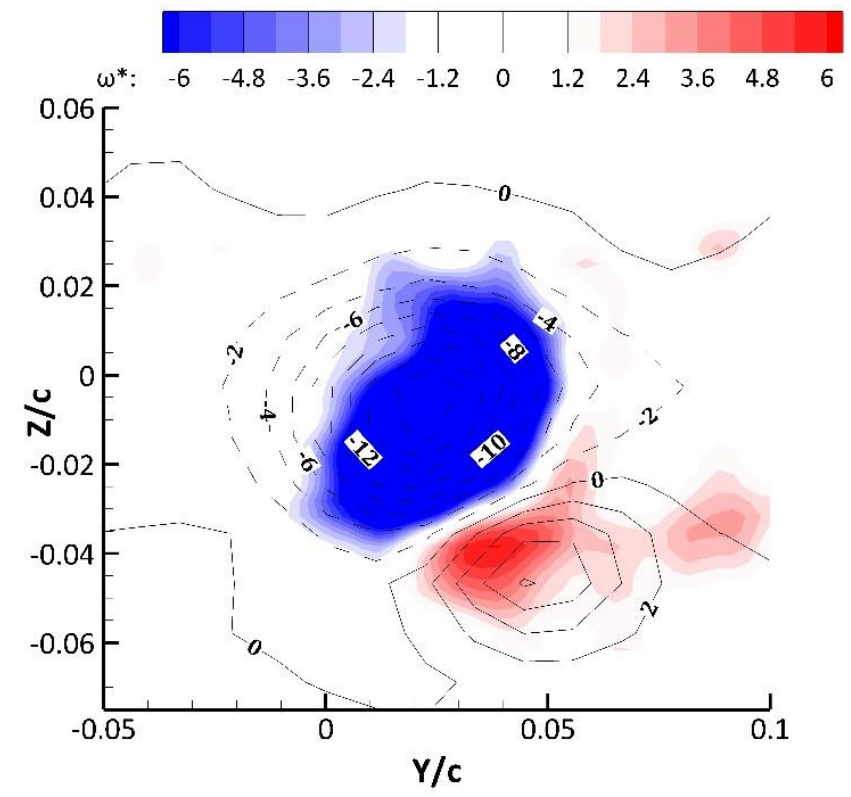

Fig 5: Non-dimensional $\mathrm{x}$-vorticity $\left(\omega^{*}=\omega_{\mathrm{x}} \mathrm{U} / \mathrm{c}\right)$ comparison of the current experiment (contour) and the SPIV experiment (lines - solid lines indicate positive vorticity and negative represents negative vorticity) carried out in Gowree et al., 2018.

\section{Conclusion}

In the present work we have studied the complex fluid dynamics of the flow around the wing of a peregrine falcon in a stoop manoeuvre using the VPIV technique. The experiments were performed in a water flume with a freestream velocity of $U=10 \mathrm{~cm} / \mathrm{s}$ and $\alpha=3.25^{\circ}$. The results demonstrate that the complicated vortices being shed from the wing tip can be accurately captured using this set-up. Comparisons made with previous SPIV studies shows that the VPIV system and associated processing algorithms, can indeed accurately and quantitatively reconstruct the unsteady and coherent vortex structures shed arising from the wing tip. The complex vortex-driven flow field seems to play a key role in helping the peregrine falcon maintain an aerodynamic advantage over its prey.

\section{Acknowledgments}


We would like to thank Prof. Christoph Bruecker of City, University of London, for allowing us to use the Falcon model and water tunnel facility for this experiment.

\section{References}

Gowree, Jagadeesh, Talboys, Lagemann, \& Brücker (2018). Vortices enable the complex aerobatics of peregrine falcons. Communications biology, 1(1), 1-7.

Selim, Gowree, Lagemann, Talboys, Jagadeesh \& Brücker (2021). The Peregrine Falcon's Dive: On the Pull-Out Maneuver and Flight Control Through Wing-Morphing. AIAA Journal, accepted awaiting proof.

Boomsma, Troolin (2018) Time-Resolved Particle Image Identification and Reconstruction for Volumetric 4D-PTV, 19th International Symposium on the Application of Laser and Imaging Techniques to Fluid Mechanics, Lisbon, Portugal July 16-19, 2018. 